\title{
Case Report: Anesthetic management for Cesarean section in a parturient with unspecified inherited bleeding disorder
}

\section{[version 1; peer review: 2 approved with reservations]}

\author{
Li Li1, Jill M. Johnsen², Chau X. Doan1, Laurent A. Bollag (iD)1 \\ ${ }^{1}$ Department of Anesthesiology \& Pain Medicine, University of Washington, Seattle, WA, 98195, USA \\ ${ }^{2}$ Department of Medicine - Hematology, University of Washington, Seattle, WA, 98195, USA
}

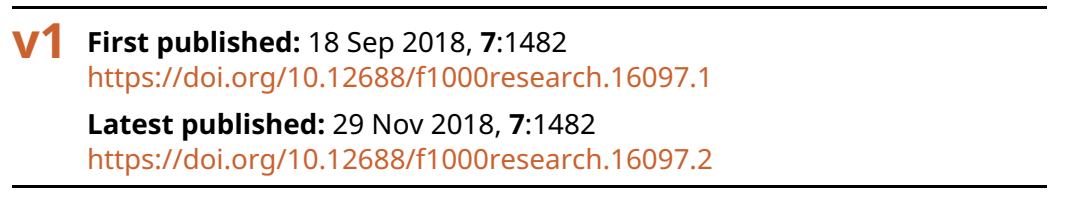

\section{Abstract}

Neuraxial anesthesia, as the standard of care for Cesarean deliveries, is associated with decreased blood loss. However, parturients with inherited bleeding disorders are at increased risk for epidural hematomas. A small retrospective study has shown that parturients with known factor deficiencies can safely undergo neuraxial anesthesia once the specific factors are replenished. We present a patient who had a considerably increased risk of peripartum bleeding from an unspecified inherited bleeding disorder and was provided a successful neuraxial anesthetic without complications. We discuss the multidisciplinary approach among the surgeons, anesthesiologists, hematologist, and nursing staff to maximize patient safety and comfort.

\section{Keywords}

Anesthesia, Spinal, Cesarean section, Parturient, Bleeding disorder

Open Peer Review
Approval Status
version 2
(revision)
29 Nov 2018
18 Sep 2018
........................................................................
1. Allison J. Lee (ID), Columbia University, New
York City, USA
2. Benjamin G. Cobb (ID), University of North
Carolina, Chapel Hill, USA
Any reports and responses or comments on the
article can be found at the end of the article.


Corresponding author: Li Li (lili.md.phd@gmail.com)

Author roles: Li L: Conceptualization, Investigation, Visualization, Writing - Original Draft Preparation, Writing - Review \& Editing; Johnsen JM: Visualization, Writing - Review \& Editing; Doan CX: Investigation, Visualization, Writing - Review \& Editing; Bollag LA: Conceptualization, Investigation, Supervision, Writing - Review \& Editing

Competing interests: No competing interests were disclosed.

Grant information: The author(s) declared that no grants were involved in supporting this work.

Copyright: $\odot 2018 \mathrm{Li}$ L et al. This is an open access article distributed under the terms of the Creative Commons Attribution License, which permits unrestricted use, distribution, and reproduction in any medium, provided the original work is properly cited.

How to cite this article: Li L, Johnsen JM, Doan CX and Bollag LA. Case Report: Anesthetic management for Cesarean section in a parturient with unspecified inherited bleeding disorder [version 1; peer review: 2 approved with reservations] F1000Research 2018, 7:1482 https://doi.org/10.12688/f1000research.16097.1

First published: 18 Sep 2018, 7:1482 https://doi.org/10.12688/f1000research.16097.1 


\section{Introduction}

Neuraxial anesthesia, which encompasses both spinal and epidural anesthesia, has been established as a safe and effective regional anesthetic technique for the obstetric patient. Although considered the standard of care for Cesarean deliveries (CDs), this technique carries a small but potentially devastating risk of neuraxial bleeding complications (e.g. spinal epidural hematomas (SEH)) that may result in significant neurological injury or compromise. The risk of SEH in obstetric patients from neuraxial anesthesia is estimated to be $1: 168,000^{1}$. Parturients with coagulopathy or bleeding diathesis are at increased risk of neuraxial hematomas ${ }^{2,3}$. Although a recent study has provided a management outline for patients with unclassified bleeding disorders undergoing procedures ${ }^{4}$, no specific recommendations were made for CDs, which is unique in that hematologic management directly impacts anesthetic management. In fact, although parturients with known hemostatic deficiencies can often safely undergo neuraxial anesthesia once the hemostatic defects are corrected $\mathrm{d}^{5-7}$, due to the lack of recommendations, neuraxial anesthesia is often avoided in these patients, as in patients with other coagulopathies ${ }^{8}$. Here, we present the anesthetic management and safe utilization of neuraxial anesthesia for a $\mathrm{CD}$ in a patient with an unspecified inherited bleeding disorder.

\section{Case description}

A 39-year-old G6P3 with an unspecified inherited bleeding disorder presented at 37-6/7 weeks gestational age for a fourth repeat scheduled $\mathrm{CD}$ in the setting of fetal macrosomia. The patient had a diagnosis of an unspecified bleeding disorder. Prior to diagnosis, her bleeding history consisted of easy bruising, gingival bleeding, heavy menstrual bleeding, and multiple post-operative hemorrhagic complications after previous surgeries. These complications included hemorrhagic compartment syndrome after an ankle surgery and bleeding complications with all three prior deliveries including persistent vaginal bleeding after her first two CDs and intra-abdominal hemorrhage after her third CD. Although she reported having had epidurals during her prior pregnancies, these anesthetic records were unavailable. Her family history is positive for mucocutaneous bleeding including maternal heavy menstrual bleeding and death of her maternal grandmother secondary to postpartum hemorrhage. Prior to this pregnancy, she was referred for evaluation of a bleeding disorder in advance of cervical spine surgery. Her extensive coagulation laboratory evaluation was unremarkable, including normal complete blood count and smear, activated partial thromboplastin time, prothrombin time, thrombin time, von Willebrand factor (VWF) parameters, fibrinogen activity, platelet aggregation studies (including ristocetin-induced platelet aggregation), platelet function assay, factor XIII level, and rotational thromboelastography. There was good documentation of her prior bleeding and no suspicion for other disorders, such as endocrine or connective tissue disorders. She was diagnosed with an unspecified inherited bleeding disorder. The differential diagnosis for her hemostatic defect included rare congenital bleeding disorders such as undetected VWF qualitative dysfunction or undetected defects in fibrin, fibrinolysis, or platelet function. She received prophylactic fresh frozen plasma (FFP), cryoprecipitate, platelets, and anti-fibrinolytic treatment as prophylaxis for her cervical spine surgery and achieved good hemostasis without complication.
Upon presentation for delivery, her laboratory values were unremarkable: hematocrit (Hct) $30 \%$, platelets $169 \times 10^{3} / \mathrm{ml}$, international normalized ratio (INR) 1.1, partial thromboplastin time (PTT) $30 \mathrm{~s}$, fibrinogen activity $461 \mathrm{mg} / \mathrm{dl}$, and a thromboelastogram (TEG) within normal parameters. Prior to her delivery, a multi-disciplinary care plan, including hematology, anesthesiology, and obstetric services was established and a delivery plan that balanced patient safety and birthing preferences agreed upon; contingency plans for transfusion and anatomic control of bleeding were in place in the event of obstetric hemorrhage. The risks and benefits of regional anesthesia were discussed at length with the patient, acknowledging the diagnostic challenges and lack of a useful laboratory monitoring test.

The patient prophylactically received 2 units of FFP, 10 units of cryoprecipitate within 4 hours prior to $\mathrm{CD}$, and 2 units of platelets immediately before the placement of spinal anesthetic. Following the delivery of a healthy infant, intravenous aminocaproic acid was immediately started and routine oxytocin was infused. Poor uterine tone was noted after delivery, which improved after single dose of 200 ug IM methylergonovine and $800 \mathrm{mg}$ buccal misoprostol administration. The estimated blood loss was 1.5 liters. In response to her higher-than-average blood loss, 2 additional units of platelets were given in the immediate postoperative period. Deep venous thrombosis prophylaxis consisted of sequential compression devices until ambulation; low molecular weight heparin was avoided. The patient was closely monitored. Her postoperative labs were notable for Hct $22 \%$, platelets $175 \times 10^{3} / \mathrm{ml}$, INR 1.1, PTT $28 \mathrm{~s}$, and fibrinogen activity $462 \mathrm{mg} / \mathrm{dl}$. Additionally, TEG results during and after the $\mathrm{CD}$ remained normal. The patient continued intravenous aminocaproic acid therapy postpartum, and transitioned to oral tranexamic acid for 5 days. She had an uneventful recovery and was discharged 3 days after surgery. The case management timeline and dosing regimen are shown in Figure 1.

\section{Discussion}

This patient's unspecified inherited bleeding disorder placed her at considerably increased risk of peripartum bleeding with risks compounded by fetal macrosomia, multiple prior CDs, and a personal history of obstetric hemorrhage in the absence of prophylaxis. Typically, for a well-defined bleeding disorder prophylaxis can be used with appropriate repletion of the respective pathway components. For patients with unspecified bleeding disorders, it is not possible to know if the hemostatic defect has been adequately corrected by the prophylaxis treatment options available. However, in this case her spine surgery history could inform a prophylaxis regimen; good communication and detailed hematology recommendations regarding the type, dose, and timing of blood product administration were critical in the planning and delivery of a safe spinal anesthetic for this patient.

Despite the potential for prolonged duration of surgery in the setting of a fourth $\mathrm{CD}$, a spinal anesthetic was preferred over a combined spinal epidural to minimize the risk of an epidural catheter-associated hematoma ${ }^{9,10}$. General anesthesia, even with comparable safety in obstetrics ${ }^{11}$, was reserved for a failed 


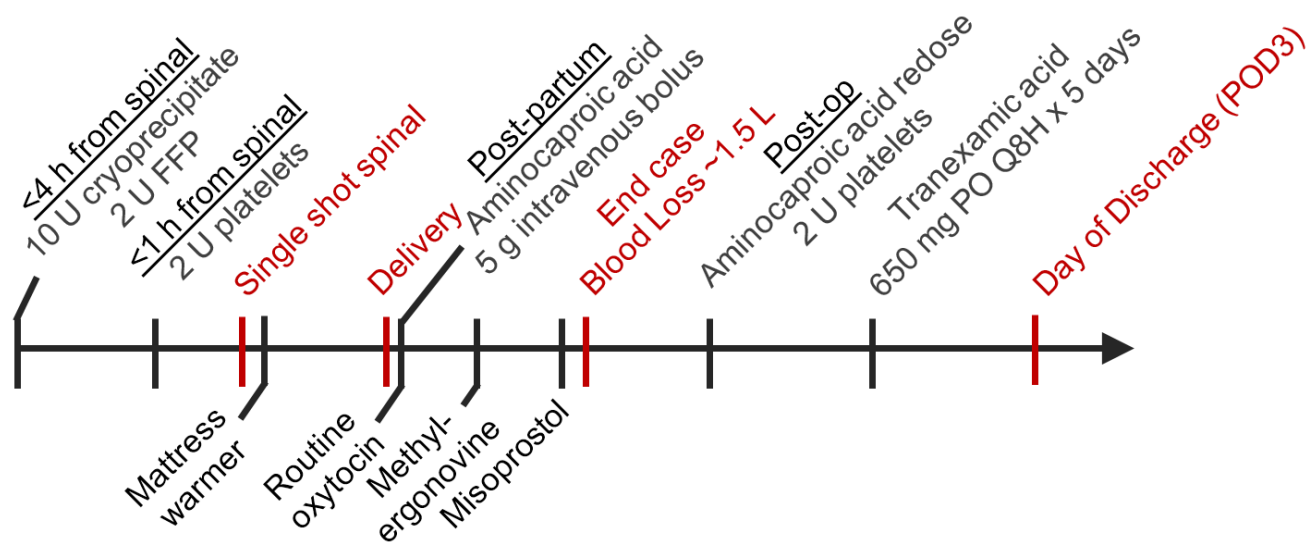

Figure 1. Case management timeline. Hematologic interventions for the planned Cesarean section are shown on top, whereas the intraoperative interventions to reduce bleeding risks are shown on the bottom of the arrow. Case events are shown in red.

spinal anesthesia or bleeding emergency to optimize maternal uterine tone ${ }^{12}$ and fetal neurological adaptation, both of which are affected by the use of volatile anesthetics, and to allow for early maternal-infant skin-to-skin bonding, honoring the mothers wish. Also important, the patient desired a regional anesthetic.

Other management considerations to guide appropriate planning and reduce the risk of significant perioperative bleeding include: early pre-anesthetic consultation, pre-operative hematology consultation, expeditious use of intraoperative uterotonics, and maintenance of normothermia to prevent hypothermic coagulopathy. Co-loading with crystalloid or colloid prior to initiation of neuraxial anesthesia can attenuate hypotensioncommonly observed with spinal-related sympathectomy-which may be exacerbated in the setting of acute hemorrhage. The use of non-steroidal anti-inflammatory drugs was avoided.

Since laboratory results, including TEG, did not reflect the patient's hemostasis defect, close intraoperative monitoring of bleeding status was performed by continually evaluating vital signs, examining blood loss in the operative field and suction canisters, and closely communicating with the obstetricians about uterine tone. The above-average blood loss was attributed to uterine atony due to observation by the surgeons, though incomplete hemostasis could not be ruled out completely. Postoperatively, close neurological exams for 24 hours to monitor for clinical signs of neuraxial hematoma were performed.

In summary, this case report highlights the importance of a multi-disciplinary approach. Close communication and coordination between the hematology, obstetric, and obstetric anesthesia services were crucial in achieving an uneventful CD in this patient with an otherwise extremely high bleeding risk.

\section{Data availability}

All data underlying the results are available as part of the article and no additional source data are required.

\section{Consent}

Written informed consent for publication of their clinical details and/or clinical images was obtained from the patient.

\section{Grant information}

The author(s) declared that no grants were involved in supporting this work.
1. Ruppen W, Derry S, McQuay H, et al.: Incidence of epidural hematoma, infection, and neurologic injury in obstetric patients with epidural analgesia/ anesthesia. Anesthesiology. 2006; 105(2): 394-9.

PubMed Abstract | Publisher Full Text

2. Moen V, Dahlgren N, Irestedt L: Severe neurological complications after central neuraxial blockades in Sweden 1990-1999. Anesthesiology. 2004; 101(4): 950-9. PubMed Abstract | Publisher Full Text

3. Gulur P, Tsui B, Pathak R, et al: Retrospective analysis of the incidence of epidural haematoma in patients with epidural catheters and abnormal coagulation parameters. Br J Anaesth. 2015; 114(5): 808-11. PubMed Abstract | Publisher Full Text

4. Obaji S, Alikhan R, Rayment R, et al: Unclassified bleeding disorders: outcome of haemostatic challenges following tranexamic acid and/or desmopressin. Haemophilia. 2016; 22(2): 285-291. PubMed Abstract | Publisher Full Text

5. Chi C, Lee CA, England A, et al.: Obstetric analgesia and anaesthesia in women with inherited bleeding disorders. Thromb Haemost. 2009; 101(6): 1104-11. PubMed Abstract | Publisher Full Text

6. Katz D, Beilin Y: Disorders of coagulation in pregnancy. Br J Anaesth. 2015; 115 Suppl 2: ii75-88.

PubMed Abstract | Publisher Full Text

7. Gonzalez-Fiol A, Eisenberger A: Anesthesia implications of coagulation and anticoagulation during pregnancy. Semin Perinatol. 2014; 38(6): 370-7. PubMed Abstract | Publisher Full Text 
8. Demers C, Derzko C, David M, et al.: No. 163-Gynaecological and Obstetric Management of Women With Inherited Bleeding Disorders. J Obstet Gynaecol Can. 2018; 40(2): e91-e103.

PubMed Abstract | Publisher Full Text

9. Pumberger M, Memtsoudis SG, Stundner O, et al:: An analysis of the safety of epidural and spinal neuraxial anesthesia in more than 100,000 consecutive major lower extremity joint replacements. Reg Anesth Pain Med. 2013; 38(6): 515-9.

PubMed Abstract | Publisher Full Text

10. Vandermeulen EP, Van Aken $\mathrm{H}$, Vermylen J: Anticoagulants and spinal-epidural anesthesia. Anesth Analg. 1994; 79(6): 1165-77.

PubMed Abstract

11. Hawkins JL, Chang J, Palmer SK, et al:: Anesthesia-related maternal mortality in the United States: 1979-2002. Obstet Gynecol. 2011; 117(1): 69-74. PubMed Abstract | Publisher Full Text

12. Yoo KY, Lee JC, Yoon MH, et al:: The effects of volatile anesthetics on spontaneous contractility of isolated human pregnant uterine muscle: a comparison among sevoflurane, desflurane, isoflurane, and halothane. Anesth Analg. 2006; 103(2): 443-7, table of contents.

Analg. 2006; 103(2): 443-7, table of contents
PubMed Abstract | Publisher Full Text 


\section{Open Peer Review}

\section{Current Peer Review Status: ? ?}

\section{Version 1}

Reviewer Report 17 October 2018

https://doi.org/10.5256/f1000research.17577.r38949

(C) 2018 Cobb B. This is an open access peer review report distributed under the terms of the Creative Commons Attribution License, which permits unrestricted use, distribution, and reproduction in any medium, provided the original work is properly cited.

\section{Benjamin G. Cobb}

Department of Anesthesiology, University of North Carolina, Chapel Hill, NC, USA

The authors describe the use of regional anesthesia for cesarean section in a parturient with an unspecified inherited bleeding disorder. In this case, the clinical bleeding history in conjunction with the hematologic lab workup leading to the diagnosis of an 'unspecified' bleeding disorder is well described. The authors highlight both the importance of early multidisciplinary collaboration in the parturient with a bleeding disorder in an effort to minimize hemorrhagic complications. In addition, the role of informed consent for a patient with an unspecified bleeding disorder and unquantifiable risk of spinal/epidural hematoma should not be understated. Additional attention to the potential limitations of factor/platelet/fibrinogen replacement in the setting of qualitative coagulation defects to prevent hemorrhagic complications of neuraxial anesthesia may be warranted in the discussion. I commend the authors for describing the often challenging anesthetic risk-benefit assessment of a patient with an unspecified bleeding disorders.

In addition to the cryoprecipitate transfusion, was von Willebrand factor/DDAVP administration considered given the unknown defect?

Please clarify the institutional oxytocin protocol post-delivery.

Following the postpartum hemorrhage, what guided the decision to transfuse two additional units of platelets postoperatively and not other factors/fibrinogen? Is there any published literature on the prevalence of 'unspecified bleeding disorders' and/or standardization of these clinical diagnoses in the setting of normal coagulation workups by hematologists?

Please clarify post-operative neurologic monitoring plan, including nursing neurological check frequency and patient surveillance counseling (concerning signs/symptoms).

Is the background of the case's history and progression described in sufficient detail? Yes

Are enough details provided of any physical examination and diagnostic tests, treatment given and outcomes? 
Yes

Is sufficient discussion included of the importance of the findings and their relevance to future understanding of disease processes, diagnosis or treatment?

Partly

Is the case presented with sufficient detail to be useful for other practitioners?

Partly

Competing Interests: No competing interests were disclosed.

I confirm that I have read this submission and believe that I have an appropriate level of expertise to confirm that it is of an acceptable scientific standard, however I have significant reservations, as outlined above.

Author Response 06 Nov 2018

Laurent Bollag, University of Washigton, Seattle, USA

Dear Dr. Cobb

Thank you for your comments to improve the quality of our manuscript.

I will reply point by point to your comments:

In addition to the cryoprecipitate transfusion, was von Willebrand factor/DDAVP administration considered given the unknown defect?

Willebrand factor/DDAVP administration was not given based on the norma; von Willebrand factor (VWF) parameters preoperatively.

Please clarify the institutional oxytocin protocol post-delivery.

Our protocol is as follows: 15 IU per hour given on a drip. Once adequate uterine tone is achieved, the dose is reduced to 7.5 IU per hour, and again, once the tone is reported to be adequate, reduced ca $50 \%$ (or $70 \mathrm{ml} / \mathrm{hr}$ ). This drip is continued postpartum.

Following the postpartum hemorrhage, what guided the decision to transfuse two additional units of platelets postoperatively and not other factors/fibrinogen?

While uterine atony was the likely reason for the $\mathrm{PPH}$, incomplete hemostasis we assumed and platelet substitution deemed the most appropriate therapeutic first step.

Is there any published literature on the prevalence of 'unspecified bleeding disorders' and/or standardization of these clinical diagnoses in the setting of normal coagulation workups by hematologists?

The lack of such literature prompted this report.

Please clarify post-operative neurologic monitoring plan, including nursing neurological check frequency and patient surveillance counseling (concerning signs/symptoms).

Local guidelines include vigilant neurological checks, including motor function of both legs 
every 4 hours for 24 hours. Patients are informed about the common symptoms of spinal nerve compression and the need to urgently report these to the nursing staff, namely:

1. rapid onset of pain

2. new onset radiculopathy

3. sensory and

4. motor function deficits

An emergent lumbar MRI will then confirm the diagnosis.

Competing Interests: None

Reviewer Report 04 October 2018

https://doi.org/10.5256/f1000research.17577.r38948

() 2018 Lee A. This is an open access peer review report distributed under the terms of the Creative Commons Attribution License, which permits unrestricted use, distribution, and reproduction in any medium, provided the original work is properly cited.

\section{Allison J. Lee}

Department of Anesthesiology, Columbia University, New York City, NY, USA

This is a description of management of a challenging clinical scenario given the non-specific diagnosis of the bleeding disorder in this patient with a desire for neuraxial anesthesia.

I was curious about the airway exam, considering that the team would have to prepare the possibility of inducing general anesthesia. I wonder if consideration was given to administering DDAVP or other treatment for von Willebrand's disease. Was an arterial line placed or considered, and what was the intravenous access in place?

I am curious about the components of the intrathecal medications for the single shot spinal anesthetic. With the possibility for prolonged surgery, were any adjuvants, such as clonidine added to prolong the anesthetic?

I would suggest editing the language in a few places. Instead of "epidurals", suggest instead, "labor epidural analgesia". Explain what is"routine oxytocin" in your institution. For what period postoperatively was the patient "closely monitored" and how? Were there nursing orders for frequent checks of neurologic status? Finally, I would suggest discussing the reasons for the low incidence of epidural hematoma in the obstetric population and including this reference: Lee $L$, et $\mathrm{al}^{1}$.

\section{References}

1. Lee LO, Bateman BT, Kheterpal S, Klumpner TT, et al.: Risk of Epidural Hematoma after Neuraxial Techniques in Thrombocytopenic Parturients: A Report from the Multicenter Perioperative Outcomes Group.Anesthesiology. 126 (6): 1053-1063 PubMed Abstract | Publisher 


\section{Full Text}

Is the background of the case's history and progression described in sufficient detail? Yes

Are enough details provided of any physical examination and diagnostic tests, treatment given and outcomes?

Partly

Is sufficient discussion included of the importance of the findings and their relevance to future understanding of disease processes, diagnosis or treatment?

Partly

Is the case presented with sufficient detail to be useful for other practitioners?

Yes

Competing Interests: No competing interests were disclosed.

I confirm that I have read this submission and believe that I have an appropriate level of expertise to confirm that it is of an acceptable scientific standard, however I have significant reservations, as outlined above.

Author Response 06 Nov 2018

Laurent Bollag, University of Washigton, Seattle, USA

Dear Dr Lee

Thank you for taking the time to review our case report to improve its quality! I addressed your comments point by point below:

I was curious about the airway exam, considering that the team would have to prepare the possibility of inducing general anesthesia. I wonder if consideration was given to administering DDAVP or other treatment for von Willebrand's disease. Was an arterial line placed or considered, and what was the intravenous access in place?

Her airway exam was as follows: Mallampati Score 2, free head and neck movement $(>6 \mathrm{~cm})$, normal mouth opening and no prominent incisors, in short pretty normal.

Willebrand factor/DDAVP administration was not recommended by the hematologist based on the normal von Willebrand factor (VWF) parameters preoperatively.

She had a well working $18 \mathrm{~g}$ IV. We decided not to place an Aline a priori, because we felt that it could be sited easily if need be. In cases where the placement is deemed technically complicated we do place A lines when a PPH is deemed likely, the same applies to a second large bore IV.

I am curious about the components of the intrathecal medications for the single shot 
spinal anesthetic. With the possibility for prolonged surgery, were any adjuvants, such as clonidine added to prolong the anesthetic?

We used out routine SPA mixture 12,5mg hyperbaric Bupivacaine, 10mcg Fentanyl and $100 \mathrm{mcg}$ PF Morphine. We did not add spinal clonidine, to avoid worsening of hypotension in a case where a PPH was deemed likely. Clonidine could also be aded later IV, to extend spinal anesthesia duration, but again potentially worsen hypotension- for hours.

Obviously, we opted not to place a CSE in this potentially coagulopathic patient.

Explain what is"routine oxytocin" in your institution. For what period postoperatively was the patient "closely monitored" and how? Were there nursing orders for frequent checks of neurologic status? Finally, I would suggest discussing the reasons for the low incidence of epidural hematoma in the obstetric population and including this reference: Lee $L$, et al1

Physiological hypercoagulability of pregnancy as well as the large epidural space in young parturients may explain why epidural hematomas in parturients are rare; additionally older guidelines are more conservative regarding platelet count cut-offs for neuraxial anesthesia. The risk of performing neuraxial anesthesia in patients with platelet counts of less than 70,000 however remains unknown.1

We have addressed your other comments in our response to reviewer 1, who raised the same questions.

\section{References}

1. Lee LO, Bateman BT, Kheterpal S, Klumpner TT, Housey M, Aziz MF, Hand KW, MacEachern M, Goodier CG, Bernstein J, Bauer ME, Multicenter Perioperative Outcomes Group Investigators: Risk of Epidural Hematoma after Neuraxial Techniques in Thrombocytopenic Parturients: A Report from the Multicenter Perioperative Outcomes Group.Anesthesiology. 126 (6): 1053-1063 PubMed Abstract | Publisher Full Text

Competing Interests: None 
The benefits of publishing with F1000Research:

- Your article is published within days, with no editorial bias

- You can publish traditional articles, null/negative results, case reports, data notes and more

- The peer review process is transparent and collaborative

- Your article is indexed in PubMed after passing peer review

- Dedicated customer support at every stage

For pre-submission enquiries, contact research@f1000.com 